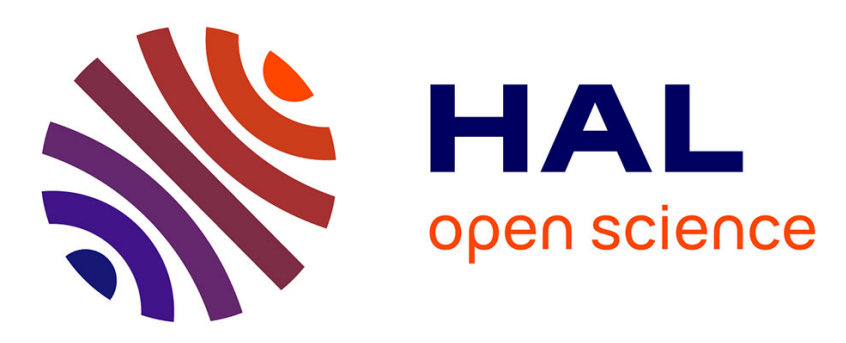

\title{
Acoustics with wall-slip flow of gas-saturated porous media
}

\author{
J. Chastanet, Pascale Royer, Jean-Louis Auriault
}

\section{To cite this version:}

J. Chastanet, Pascale Royer, Jean-Louis Auriault. Acoustics with wall-slip flow of gassaturated porous media. Mechanics Research Communications, 2004, 31 (3), pp.277-286. 10.1016/j.mechrescom.2003.10.003 . hal-01715870

\section{HAL Id: hal-01715870 https://hal.science/hal-01715870}

Submitted on 23 Feb 2018

HAL is a multi-disciplinary open access archive for the deposit and dissemination of scientific research documents, whether they are published or not. The documents may come from teaching and research institutions in France or abroad, or from public or private research centers.
L'archive ouverte pluridisciplinaire HAL, est destinée au dépôt et à la diffusion de documents scientifiques de niveau recherche, publiés ou non, émanant des établissements d'enseignement et de recherche français ou étrangers, des laboratoires publics ou privés. 


\title{
Acoustics with wall-slip flow of gas-saturated porous media
}

\author{
J. Chastanet ${ }^{\mathrm{a}, *}$, P. Royer ${ }^{\text {a }}$, J.-L. Auriault ${ }^{\text {a }}$ \\ "Laboratoire "Sols, Solides, Structures" (3S), UJF, INPG, CNRS, BP 53, 38041 Grenoble Cedex, France
}

\begin{abstract}
This work is aimed at deriving and at analyzing the dynamic filtration law that describes the acoustics of a gas saturated porous medium, when a wall-slip flow occurs due to low gas pressure. The dynamic filtration law is derived by upscaling the pore-scale description that consists of the equations of linear acoustics and a wall-slip condition on the pore wall. We have shown that the effective dynamic permeability depends on the Knudsen number and can be approximated by the generalized Klinkenberg's law at low Knudsen numbers. Analysis of these results on a simple geometry proves that this approximation fails at reproducing the macroscopic inertial effects at high pulsations.
\end{abstract}

Keywords: Acoustics; Klinkenberg's effect; Knudsen; Gas flow; Low pressure; Wall-slip flow; Dynamic permeability; Upscaling; Homogenisation

\section{Introduction}

The objective of this work is to derive the dynamic filtration law that describes the acoustics of a rigid porous medium saturated by a gas when a wall-slip flow occurs on the pore wall due to low gas pressure. Our purpose is to use an upscaling technique, namely the homogenisation method of multiple scale expansions (Benssoussan et al., 1978; Sanchez-Palencia, 1980), so as to derive the macroscopic dynamic filtration law by starting from the pore-scale description; this latter consists of the equations of linear acoustics for the fluid and a wall-slip condition on the solid/fluid interface.

At constant pulsation $\omega$, homogenisation of the pore-scale description that consists of the equations of linear acoustics and the no-slip condition on the pore wall leads to the generalized Darcy' law (Biot, 1956; Levy, 1979; Auriault, 1980; Burridge and Keller, 1981):

$$
\boldsymbol{v}=-\widetilde{K}(\omega) \nabla p
$$

in which the dynamic permeability tensor is complex-valued, pulsation dependent and inversible:

$$
\widetilde{K}^{-1}=\widetilde{H}=\widetilde{H}_{1}+\mathrm{i} \widetilde{H}_{2} .
$$

The generalized Darcy's law can thus be rewritten as follows:

\footnotetext{
* Corresponding author. Fax: +33-4-76-82-70-43.

E-mail address: juliette.chastanet@hmg.inpg.fr (J. Chastanet).
} 


$$
\boldsymbol{\nabla}(\phi p)=-\phi \widetilde{H}_{1} v-\underset{\omega}{\stackrel{\phi}{\dot{\boldsymbol{v}}}} \widetilde{H}
$$

where $\phi$ represents the porosity. This writing expresses a momentum balance in which the viscous term $\phi \widetilde{H}_{1} v$ characterizes the dissipation and the inertial term $\frac{\phi}{\omega} \widetilde{H}_{2} \dot{v}$ is related to the effective fluid density.

At low gas pressure, the no-slip condition on the pore wall may break down. The wall-slip flow is due to the fact that the mean free path of a molecule $\lambda$, i.e. the mean distance that it travels before it collides with another molecule, is inversely proportional to the pressure:

$$
\lambda=\frac{\mu}{p} \sqrt{\pi R T / 2 M}
$$

Hence, at low gas pressure the mean free path may become comparable with the pore size $l$. In other words, the Knudsen number may reach the order of one:

$$
K_{n}=\frac{\lambda}{l} \leqslant \mathrm{O}(1) .
$$

As a result, gas molecules diffuse on the pore wall: a wall-slip flow occurs. This phenomenon, which has widely been investigated in quasi-statics is called Klinkenberg's effect. At the sample scale, it leads to important deviations from Darcy's law. For describing the phenomenon at the sample scale, Klinkenberg (1941) proposed to replace the permeability in Darcy's law by a pressure-dependent gas permeability:

$$
k_{\mathrm{g}}=k_{1}\left(1+4 c K_{n}\right)=k_{1}\left(1+\frac{b}{p}\right),
$$

in which $k_{1}$ denotes the liquid permeability and where $c$ and $b$ are constants. In Skjetne and Auriault (1999), a tensorial form of Klinkenberg's law (3) is derived by homogenisation by considering a quasi-static wallslip flow at the pore scale.

The question of the dynamic filtration law when a local wall-slip flow occurs has not yet been addressed. This investigation by homogenisation consists of the extensions of Auriault (1980) to the local wall-slip flow problem and of Skjetne and Auriault (1999) to the acoustic regime. One of the issues is thus to determine whether there exists a generalized Klinkenberg's law, i.e. whether the dynamic permeability could be such as:

$$
\widetilde{K}_{\mathrm{g}}=\widetilde{K}_{1}(\omega)\left[1+K_{n} \widetilde{c}(\omega)\right] .
$$

The paper is organized in four parts. Firstly in Section 2, we briefly introduce the homogenisation method. Then, in Section 3, we analyze the pore scale gas wall-slip flow problem. Section 4 is devoted to the homogenisation of this local description. The general dynamic filtration law is derived by considering a Knudsen number of order of one. As in Skjetne and Auriault (1999), a perturbation analysis in terms of $K_{n}$ is then carried out in Section 5 to obtain an approximation $K_{\text {app }}$ of the general dynamic filtration law $K_{\text {eff }}$ at low Knudsen number (4). Finally, in Section 6, we examine our results on the simple geometry of a bundle of capillaries. We show that the wall-slip entails a decrease in both the dissipation and the inertial terms. The comparison of $\widetilde{K}_{\text {eff }}$ with $\widetilde{K}_{\text {app }}$ shows that approximating $\widetilde{K}_{\text {eff }}$ by $\widetilde{K}_{\text {app }}$ at low Knudsen numbers is only appropriate at low pulsations.

\section{Homogenisation method}

The essence of homogenisation techniques is to determine an equivalent macroscopic behaviour by upscaling the local description. The fundamental assumption behind any homogenisation method is that the scales must be separated. This condition is expressed as $l \ll L$, where $l$ and $L$ are the characteristic 
lengths at the heterogeneity scale and the macroscopic scale, respectively. This definition conjures up a purely geometric separation of scales, but this fundamental condition must also be satisfied by the physical process considered. For wave propagation in a heterogeneous medium, the heterogeneity characteristic length $l$ must also be small compared to the wavelength.

We use the homogenisation method for periodic structures (also called method of multiple scale expansions) introduced by Benssoussan et al. (1978) and Sanchez-Palencia (1980). The key parameter of the method is the scale ratio:

$$
\varepsilon=\frac{l}{L} \ll 1 \text {. }
$$

With this homogenisation method, the medium is also assumed to be periodic. This assumption is actually not a restriction; it allows derivation of the macroscopic model without any assumption on the form of the macroscopic equations. In this study, we use the methodology suggested in Auriault (1991), with which the problem is tackled in a more physical manner. This formulation uses the dimensionless numbers that arise from the local description. These dimensionless numbers must be estimated with respect to the scale ratio $\varepsilon$.

Using the two characteristic lengths, two dimensionless space variables are defined:

$$
\boldsymbol{y}=\frac{X}{l}, \quad \boldsymbol{x}=\frac{X}{L},
$$

where $\boldsymbol{X}$ is the physical space variables. If the condition of separation of scales is satisfied, then $\boldsymbol{y}$ and $\boldsymbol{x}$ appear as two independent space variables: $\boldsymbol{y}$ is the microscopic variable and describes the heterogeneity scale, whereas $\boldsymbol{x}$ is the macroscopic variable. As a consequence, the physical variables of the problem, $p, \rho$ and $\boldsymbol{v}$, are a priori functions of $\boldsymbol{y}$ and $\boldsymbol{x}$ :

$$
p=p(\boldsymbol{y}, \boldsymbol{x}), \quad \rho=\rho(\boldsymbol{y}, \boldsymbol{x}), \quad \boldsymbol{v}=\boldsymbol{v}(\boldsymbol{y}, \boldsymbol{x}) .
$$

The homogenisation method of multiple scale is based on the fundamental premise that if the scales are well separated, then all physical variables can be looked for in the form of asymptotic expansions in powers of $\varepsilon$ :

$$
\begin{aligned}
& p=p^{(0)}(\boldsymbol{x}, \boldsymbol{y})+\varepsilon p^{(1)}(\boldsymbol{x}, \boldsymbol{y})+\cdots \\
& \rho=\rho^{(0)}(\boldsymbol{x}, \boldsymbol{y})+\varepsilon \rho^{(1)}(\boldsymbol{x}, \boldsymbol{y})+\cdots \\
& \boldsymbol{v}=\boldsymbol{v}^{(0)}(\boldsymbol{x}, \boldsymbol{y})+\varepsilon \boldsymbol{v}^{(1)}(\boldsymbol{x}, \boldsymbol{y})+\cdots
\end{aligned}
$$

in which the functions $p^{(i)}, \rho^{(i)}$ and $\boldsymbol{v}^{(i)}$ are $\boldsymbol{y}$-periodic. The method consists of incorporating expansions (6)(8) in the dimensionless form of the local description. Solving the boundary-value problems arising at the successive orders of $\varepsilon$ leads to the macroscopic description.

\section{Pore-scale gas wall-slip flow problem}

We consider a periodic rigid porous medium saturated by a gas at low pressure. Let us denote by $\Omega$ the period and by $l$ its scale length, which is small compared to a typical macroscopic length $L$. Within the period, $\Omega_{\mathrm{p}}$ and $\Omega_{\mathrm{s}}$ represent the fluid and solid parts of the period, respectively, and $\Gamma$ denotes the pore surface (Fig. 1).

We shall consider isothermal motions of the fluid which are small enough to be governed by the linearized Navier-Stokes equations. In order to account for a possible Klinkenberg's effect, we consider a wallslip condition on $\Gamma$. For time harmonic motions with angular frequency $\omega$, these equations are

$$
\mu \boldsymbol{\nabla}^{2} \boldsymbol{v}+(\eta+\mu) \boldsymbol{\nabla}(\boldsymbol{\nabla} \cdot \boldsymbol{v})-\nabla p=\mathrm{i} \omega \rho^{\mathrm{e}} \boldsymbol{v} \quad \text { in } \Omega_{\mathrm{p}},
$$




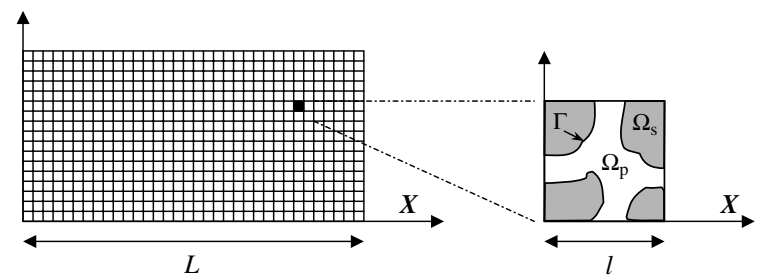

(a)

(b)

Fig. 1. Two-scale medium: (a) macroscopic sample, (b) periodic cell.

$$
\begin{aligned}
& \mathrm{i} \omega \rho+\rho^{\mathrm{e}} \boldsymbol{\nabla} \cdot \boldsymbol{v}=0 \quad \text { in } \Omega_{\mathrm{p}}, \\
& p=A \rho(\mathrm{A} \text { is a constant }) \quad \text { in } \Omega_{\mathrm{p}}, \\
& \boldsymbol{v}=-c \lambda \boldsymbol{t}_{\mathbf{1}} \cdot(\boldsymbol{\nabla} \boldsymbol{v}) \cdot \boldsymbol{n \boldsymbol { t } _ { 1 }} \quad \text { on } \Gamma .
\end{aligned}
$$

In the above equations, $\mu$ and $\eta$ denote the viscosities, $\rho^{\mathrm{e}}$ represents the density at equilibrium, $\boldsymbol{v}, p$ and $\rho$ denote the fluid velocity, the pressure and the density perturbations, respectively, and $c$ is a constant. Vectors $\boldsymbol{t}_{\mathbf{1}}$ and $\boldsymbol{n}$ represent the unit vectors tangential and normal to $\Gamma$, respectively.

From the above local description (9)-(12) arise the four following dimensionless numbers:

$$
Q=\frac{|\boldsymbol{\nabla} p|}{\left|\mu \boldsymbol{\nabla}^{2} \boldsymbol{v}\right|}, \quad R_{t}=\frac{\left|\rho^{\mathrm{e}} \frac{\partial \boldsymbol{v}}{\partial t}\right|}{\left|\mu \boldsymbol{\nabla}^{2} \boldsymbol{v}\right|}, \quad S=\frac{\left|\frac{\partial \rho}{\partial t}\right|}{\left|\rho^{\mathrm{e}} \boldsymbol{\nabla} \cdot \boldsymbol{v}\right|}, \quad B=\frac{\left|-c \lambda \boldsymbol{t}_{1} \cdot(\boldsymbol{\nabla} \boldsymbol{v}) . \boldsymbol{n} \boldsymbol{t}_{\mathbf{1}}\right|}{|\boldsymbol{v}|} .
$$

We shall now relate the order of magnitude of these dimensionless numbers to the magnitude of $\varepsilon$. We arbitrarily choose the local scale length $l$ as the reference characteristic length for estimating the differential operators. The dimensionless number $Q$ is the ratio of the pressure gradient to the viscous forces. It can be shown that $Q_{l}=\mathrm{O}\left(\varepsilon^{-1}\right)$ (Auriault, 1980). The transient Reynolds number, $R_{t}$, is the ratio of the transient term to the viscous term. Under acoustic perturbations the inertial term does act at the local scale, which reads $R_{t_{l}}=\mathrm{O}(1)$. The Strouhal number, $S$, characterizes the transient behaviour of flow and a macroscopic transient behaviour corresponds to $S_{L}=\mathrm{O}(1)$ which gives $S_{l}=\mathrm{O}(\varepsilon)$. The reader is referred to Auriault (1980) for details on the estimation of $Q, R_{t}$ and $S$. Now, the parameter $B$ is a measure of the wall-slip effect. As $c=\mathrm{O}(1)$ (Cercignani, 1988), we obtain

$$
B=\mathrm{O}\left(K_{n}\right) \text {. }
$$

The dimensionless writing of the local description (9)-(12) is therefore the following, in which all quantities are now dimensionless quantities (for ease of formulation we have kept, for the dimensionless quantities, the same notations as for their physical counterparts):

$$
\begin{aligned}
& \mu \nabla^{2} \boldsymbol{v}+(\eta+\mu) \nabla(\nabla \cdot v)-\varepsilon^{-1} \nabla p=\mathrm{i} \omega \rho^{\mathrm{e}} \boldsymbol{v} \text { in } \Omega_{\mathrm{p}}, \\
& \varepsilon i \omega \rho+\rho^{\mathrm{e}} \boldsymbol{\nabla} \cdot \boldsymbol{v}=0 \quad \text { in } \Omega_{\mathrm{p}}, \\
& p=A \rho \quad \text { in } \Omega_{\mathrm{p}}, \\
& \boldsymbol{v}=-K_{n} \boldsymbol{t}_{\mathbf{1}} \cdot(\boldsymbol{\nabla} \boldsymbol{v}) \cdot \boldsymbol{n} \boldsymbol{t}_{\mathbf{1}} \quad \text { on } \Gamma .
\end{aligned}
$$

The dimensionless gradient operator is given by

$$
\boldsymbol{\nabla}=\nabla_{y}+\varepsilon \nabla_{x},
$$


where $\nabla_{y}$ and $\nabla_{x}$ are the gradient operators with respect to $\boldsymbol{y}$ and $\boldsymbol{x}$, respectively. The purpose of the next section is the derivation of the macroscopic description when $K_{n}=\mathrm{O}(1)$, which means that: $\varepsilon \ll K_{n} \ll \varepsilon^{-1}$. The subcase defined by $\varepsilon \ll K_{n} \ll 1$ will then be considered in Section 5 .

\section{Derivation of the dynamic filtration law}

We now consider the case $K_{n}=\mathrm{O}(1)$ and apply the homogenisation procedure by incorporating the asymptotic expansions (6)-(8) in the dimensionless local description (13)-(16).

The lowest order of Eqs. (13) and (15) yields

$$
p^{(0)}=p^{(0)}(\boldsymbol{x}, t) \quad \text { and } \quad \rho^{(0)}=\rho^{(0)}(\boldsymbol{x}, t) .
$$

Considering the next order, we obtain a boundary-value problem of unknowns $\boldsymbol{v}^{(\mathbf{0})}$ and $p^{(1)}$ :

$$
\begin{aligned}
& \nabla_{y}^{2} \boldsymbol{v}^{(\mathbf{0})}-\nabla_{x} p^{(0)}-\nabla_{y} p^{(1)}=\mathrm{i} \omega \rho^{\mathrm{e}} \boldsymbol{v}^{(\mathbf{0})} \text { in } \Omega_{\mathrm{p}}, \\
& \boldsymbol{\nabla}_{y} \cdot \boldsymbol{v}^{(\mathbf{0})}=0 \quad \text { in } \Omega_{\mathrm{p}}, \\
& \boldsymbol{v}^{(\mathbf{0})}=-K_{n} \boldsymbol{t}_{\mathbf{1}}^{(\mathbf{0})} \cdot \nabla_{y} \boldsymbol{v}^{(\mathbf{0})} \cdot \boldsymbol{n} \boldsymbol{t}_{\mathbf{1}}^{(\mathbf{0})} \quad \text { on } \Gamma,
\end{aligned}
$$

where $\boldsymbol{v}^{(\mathbf{0})}$ and $p^{(1)}$ are $\Omega$-periodic.

The solution to this system may be written as follows:

$$
\boldsymbol{v}^{(\mathbf{0})}=-\tilde{k}\left(\boldsymbol{y}, K_{n}, \omega\right) \boldsymbol{\nabla}_{x} p^{(0)}
$$

Its average over the period yields:

$$
\left\langle\boldsymbol{v}^{(\mathbf{0})}\right\rangle=-\widetilde{K}_{\mathrm{eff}}\left(K_{n}, \omega\right) \boldsymbol{\nabla}_{x} p^{(0)}, \quad \widetilde{K}_{\text {eff }}=\left\langle\tilde{k}\left(\boldsymbol{y}, K_{n}, \omega\right)\right\rangle,
$$

where $\langle\cdot\rangle$ denotes the average over the period and is defined by:

$$
\langle\cdot\rangle=\frac{1}{|\Omega|} \int_{\Omega_{\mathrm{p}}} \cdot \mathrm{d} \Omega .
$$

Eq. (20) is the generalized filtration law. It differs from the generalized Darcy's law (1) in the fact that the effective dynamic permeability tensor $\widetilde{K}_{\text {eff }}$ depends upon the Knudsen number.

\section{Low Knudsen number approximation: generalized Klinkenberg's law}

In the above derivation of the macroscopic filtration law, we have considered $K_{n}=\mathrm{O}(1)$, that is $\varepsilon \ll K_{n} \ll \varepsilon^{-1}$. In order to get more insight into the expression of $\widetilde{K}_{\text {eff }}$, let now consider the case of low Knudsen number:

$$
\varepsilon \ll K_{n} \ll 1 \text {. }
$$

As in Skjetne and Auriault (1999), we now look for $\boldsymbol{v}^{(\mathbf{0})}$ and $p^{(1)}$ in the form of asymptotic expansions of $K_{n}$ :

$$
\begin{aligned}
& \boldsymbol{v}^{(\mathbf{0})}=\boldsymbol{v}^{\mathbf{0}}+K_{n} \boldsymbol{v}^{\mathbf{1}}+\left(K_{n}\right)^{2} \boldsymbol{v}^{\mathbf{2}}+\cdots \\
& p^{(1)}=p^{0}+K_{n} p^{1}+\left(K_{n}\right)^{2} p^{2}+\cdots
\end{aligned}
$$

Substituting the above expansions into boundary-value problem (17)-(19) yields at the first order: 


$$
\begin{aligned}
& \nabla_{y}^{2} \boldsymbol{v}^{\mathbf{0}}-\nabla_{x} p^{(0)}-\nabla_{y} p^{0}=\mathrm{i} \omega \rho^{\mathrm{e}} \boldsymbol{v}^{\mathbf{0}} \text { in } \Omega_{\mathrm{p}}, \\
& \boldsymbol{\nabla}_{y} \cdot \boldsymbol{v}^{\mathbf{0}}=0 \quad \text { in } \Omega_{\mathrm{p}}, \\
& \boldsymbol{v}^{\mathbf{0}}=0 \quad \text { on } \Gamma .
\end{aligned}
$$

The solution to this linear well-posed problem is:

$$
\boldsymbol{v}^{\mathbf{0}}=-\tilde{k}^{0}(\boldsymbol{y}, \omega) \boldsymbol{\nabla}_{x} p^{(0)}
$$

and its average over the period is written as:

$$
\left\langle\boldsymbol{v}^{\mathbf{0}}\right\rangle=-\widetilde{K}^{0}(\omega) \nabla_{x} p^{(0)},
$$

where

$$
\widetilde{K}^{0}=\left\langle\tilde{k}^{0}\right\rangle .
$$

$\widetilde{K}^{0}$ is the dynamic permeability tensor in the absence of wall-slip flow.

At the second order, we obtain the following wall-slip correction problem:

$$
\begin{aligned}
& \nabla_{y}^{2} \boldsymbol{v}^{\mathbf{1}}-\nabla_{y} p^{1}=\mathrm{i} \omega \rho^{\mathrm{e}} \boldsymbol{v}^{\mathbf{1}} \text { in } \Omega_{\mathrm{p}}, \\
& \boldsymbol{\nabla}_{y} \cdot \boldsymbol{v}^{\mathbf{1}}=0 \quad \text { in } \Omega_{\mathrm{p}}, \\
& \boldsymbol{v}^{\mathbf{1}}=-\boldsymbol{t}_{\mathbf{1}}^{(\mathbf{0})} \cdot \nabla_{y} \boldsymbol{v}^{\mathbf{0}} \cdot \boldsymbol{n t _ { \mathbf { 1 } } ^ { ( \mathbf { 0 } ) }} \text { on } \Gamma .
\end{aligned}
$$

The average over the period of the solution $\boldsymbol{v}^{1}$ to the above well-posed problem is written as:

$$
\left\langle\boldsymbol{v}^{1}\right\rangle=-\widetilde{K}^{1}(\omega) \nabla_{x} p^{(0)},
$$

in which $\widetilde{K}^{1}$ is a complex permeability tensor.

According to (26) and (30), the average of expansion (21) up to the second order leads to:

$$
\left\langle\boldsymbol{v}^{(\mathbf{0})}\right\rangle=-\left(\widetilde{K}^{0}+K_{n} \widetilde{K}^{1}\right) \nabla_{x} p^{(0)}
$$

which can also be written as:

$$
\begin{aligned}
\left\langle\boldsymbol{v}^{(\mathbf{0})}\right\rangle & =-\widetilde{K}_{\text {app }} \nabla_{x} p^{(0)}, \\
\widetilde{K}_{\text {app }} & =\widetilde{K}^{0}(\omega)\left(1+K_{n} \widetilde{C}(\omega)\right) .
\end{aligned}
$$

It appears that the structure of Eq. (33) is similar to Klinkenberg's law (4). Tensors $\widetilde{K}^{0}$ and $\widetilde{C}$ are complex and pulsation dependent. When taking $\omega=0$, the inertial terms in systems (23)-(25) and (27)-(29) vanish and $\widetilde{K}_{\text {app }}$ is then the Klinkenberg gas permeability. When written in time space, Eq. (32) would display a convolution product which characterizes memory effects. These effects are not due to the wall-slip flow as they already exist in the absence of the wall-slip (Auriault, 1980).

In order to illustrate the above results, to examine the influence of the wall-slip effect upon the dynamic filtration law and to compare $\widetilde{K}_{\text {eff }}$ and $\widetilde{K}_{\text {app }}$, we now consider the problem on the simple geometry of a bundle of capillaries. 


\section{Results in a bundle of capillaries}

\subsection{Dynamic filtration law}

The purpose of this section is to examine the macroscopic law for the acoustics with wall-slip effects of a bundle of capillaries of radius $a$ saturated by a gas. According to the results obtained in Section 4, the macroscopic behaviour is described by

$$
\left\langle v^{(0)}\right\rangle=-K_{\mathrm{eff}}\left(K_{n}, \omega\right) \frac{\mathrm{d} p^{(0)}}{\mathrm{d} x} .
$$

The dynamic permeability $K_{\text {eff }}$ is defined by

$$
K_{\text {eff }}=\frac{\phi}{\pi a^{2}} \int_{0} 2 \pi r k\left(r, K_{n}, \omega\right) \mathrm{d} r
$$

where $k$ is such that

$$
v^{(0)}=-k \frac{\mathrm{d} p^{(0)}}{\mathrm{d} x}
$$

In a single capillary, the local boundary-value problem (17)-(19) reduces to:

$$
\begin{aligned}
& \frac{\mathrm{d}^{2} v^{(0)}}{\mathrm{d} r^{2}}+\frac{1}{r} \frac{\mathrm{d} v^{(0)}}{\mathrm{d} r}-\frac{\mathrm{i} \omega \rho^{\mathrm{e}}}{\mu} v^{(0)}=\frac{1}{\mu} \frac{\mathrm{d} p^{(0)}}{\mathrm{d} x}, \\
& \frac{1}{\mu} \frac{\mathrm{d} p^{(1)}}{\mathrm{d} r}=0, \\
& v^{(0)}(a)=-c \lambda \frac{\mathrm{d} v^{(0)}}{\mathrm{d} r} .
\end{aligned}
$$

Solving the above boundary-value problem and then using (35) and (34) yields:

$$
K_{\mathrm{eff}}=-\frac{\phi}{\mathrm{i} \omega \rho^{\mathrm{e}}} \frac{J_{2}(a \alpha)+K_{n} a \alpha J_{1}(a \alpha)}{J_{0}(a \alpha)-K_{n} a \alpha J_{1}(a \alpha)},
$$

where $J_{i}$ are Bessel functions and $\alpha=\mathrm{i} \sqrt{\mathrm{i} \omega \rho^{\mathrm{e}} / \mu}$. It can be seen from Eq. (39) that the generalized Klinkenberg's law (4) is not verified. Let define the inverse permeability by $K_{\text {eff }}^{-1}=H_{\text {eff }}=H_{1}+\mathrm{i} H_{2}$. The term $H_{1}$ represents the dissipation whereas $H_{2} \omega^{-1}$ is the inertial term. The curves of $H_{1}$ and $H_{2} \omega^{-1}$ with respect to the dimensionless pulsation $\omega^{*}=\omega \rho^{\mathrm{e}} a^{2} / \mu$ are plotted in Fig. 2 for different values of $K_{n}$. When $K_{n}=0$, the profiles of both curves are those obtained in the case of acoustics without Klinkenberg's effect: the inertial term tends towards 1 whereas the dissipation term increases with the pulsation. The enhanced value of $\mathrm{H}_{2} \mathrm{\omega}^{-1}$ (i.e. greater than 1) at low pulsation is due to the added mass effect. When the Knudsen number is increased, both the dissipative and the inertial terms decrease as a result of the increase of the wall-slip effect.

\subsection{Generalized Klinkenberg's law}

We now consider the case $\varepsilon \ll K_{n} \ll 1$ (Section 5). The macroscopic law reads

$$
\left\langle v^{(0)}\right\rangle=-K_{\text {app }}(\omega) \frac{\mathrm{d} p^{(0)}}{\mathrm{d} x},
$$




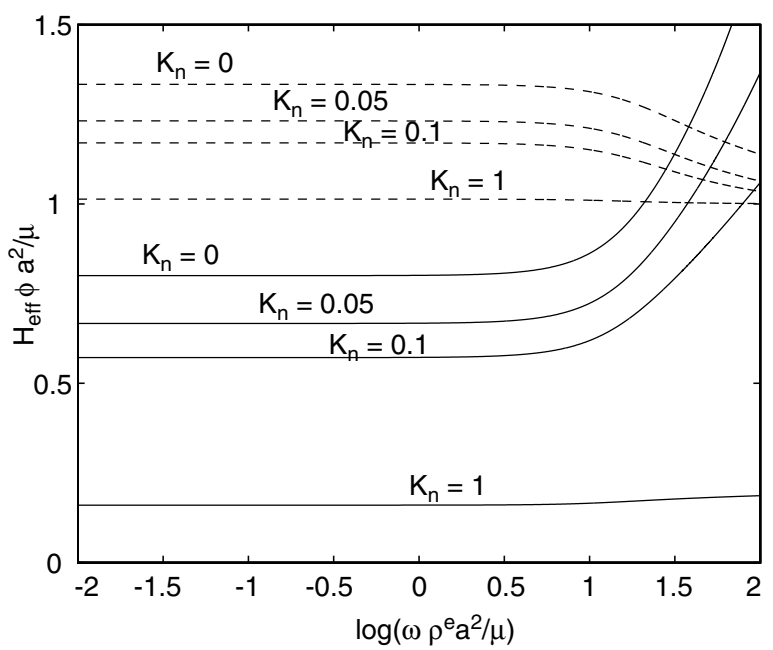

Fig. 2. Variations of $H_{\mathrm{eff}}=H_{1}+\mathrm{i} H_{2}$ with respect to the dimensionless pulsation for distinct values of the Knudsen number. Plain line: $0.1 H_{1}$ (dissipative term); dashed line: $\omega^{-1} H_{2}$ (inertial term).

where

$$
K_{\text {app }}=K^{0}+K_{n} K^{1} .
$$

The expression of $K_{\text {app }}$ may be obtained by either solving both systems (23)-(25) and (27)-(29) for determining $K^{0}$ and $K^{1}$, respectively, or by considering a limited expansion of $K_{\text {eff }}$ for low values of $K_{n}$. We thus obtain:

$$
K_{\mathrm{app}}=-\frac{\phi}{\mathrm{i} \omega \rho}\left(\frac{J_{2}(a \alpha)}{J_{0}(a \alpha)}+K_{n} \frac{2 J_{1}^{2}(a \alpha)}{J_{0}^{2}(a \alpha)}\right) .
$$

This expression for $K_{\text {app }}$ allows us to evaluate how $K_{\text {eff }}$ deviates from the generalised Klinkenberg's law. It can be shown that:

$$
\frac{K_{\mathrm{eff}}}{K^{0}}=\frac{K_{\mathrm{app}}}{K^{0}}+K_{n}^{2} a \alpha \frac{K^{1}}{K^{0}}\left(\frac{J_{1}(a \alpha)}{J_{0}(a \alpha)-K_{n} a \alpha J_{1}(a \alpha)}\right) .
$$

The curves of the real and imaginary parts of $H_{\text {eff }}=1 / K_{\text {eff }}$ and $H_{\text {app }}=1 / K_{\text {app }}$ with respect to the $\log$ of the dimensionless pulsation $\omega^{*}$ are plotted in Fig. 3, for different values of the Knudsen number. At low Knudsen number (Fig. 3a), the dissipation term (i.e. the real part) of $H_{\text {app }}$ is in good agreement with that of $H_{\text {eff }}$, as long as $\log \omega^{*} \leqslant 1$, whereas the inertial term is slightly underestimated for any value of the pulsation. For $K_{n}=0.5$ (Fig. 3b), both the real and imaginary parts of $H_{\text {app }}$ strongly differ from those of $H_{\text {eff }}$. In particular, the fact that the imaginary part is lower than one, which is physically meaningless, proves that the approximation $H_{\text {app }}$ for $H_{\text {eff }}$ (resp. $K_{\text {app }}$ for $K_{\text {eff }}$ ) is no longer valid.

Fig. 4 shows the plots of the real and imaginary parts of $H_{\text {eff }}$ and $H_{\text {app }}$ with respect to the Knudsen number, for two values of the dimensionless pulsation $\left(\omega^{*}=0.05\right.$ and $\left.\omega^{*}=5\right)$. The real parts of $H_{\text {app }}$ and $H_{\text {eff }}$ perfectly coincide for both values of the pulsation (Fig. 4a), whereas the imaginary parts strongly differ as soon as $K_{n}$ is greater than 0.05 .

Therefore, on this simple geometry, the low Knudsen number approximation $K_{\text {app }}$ of the dynamic permeability $K_{\text {eff }}$ is valid when $K_{n} \leqslant 0.05$ and $\log \omega^{*} \leqslant 1$. 

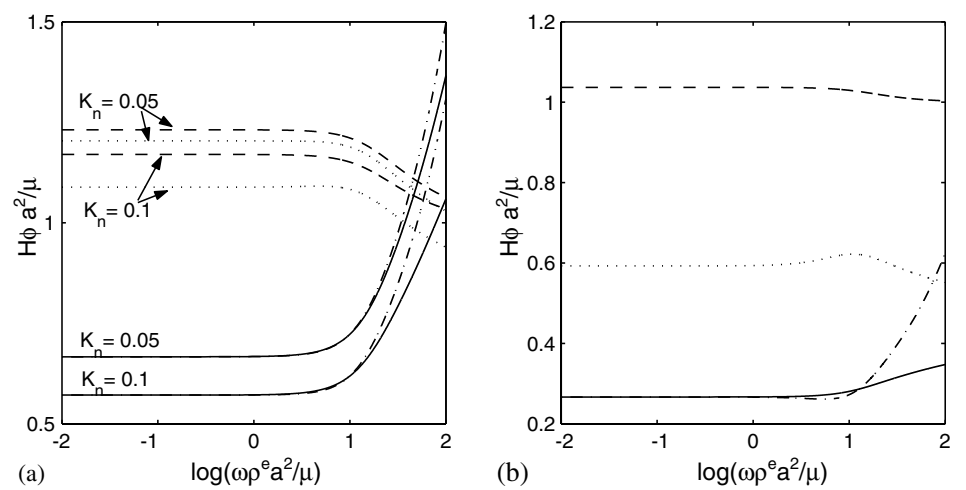

Fig. 3. Variations of $H_{\text {eff }}$ (real part in plain line and imaginary part in dashed line) and $H_{\text {app }}$ (real part in dash-dot line and imaginary part in dot line) with respect to the dimensionless pulsation: (a) for distinct low values of the Knudsen number; (b) for $K_{n}=0.5$.
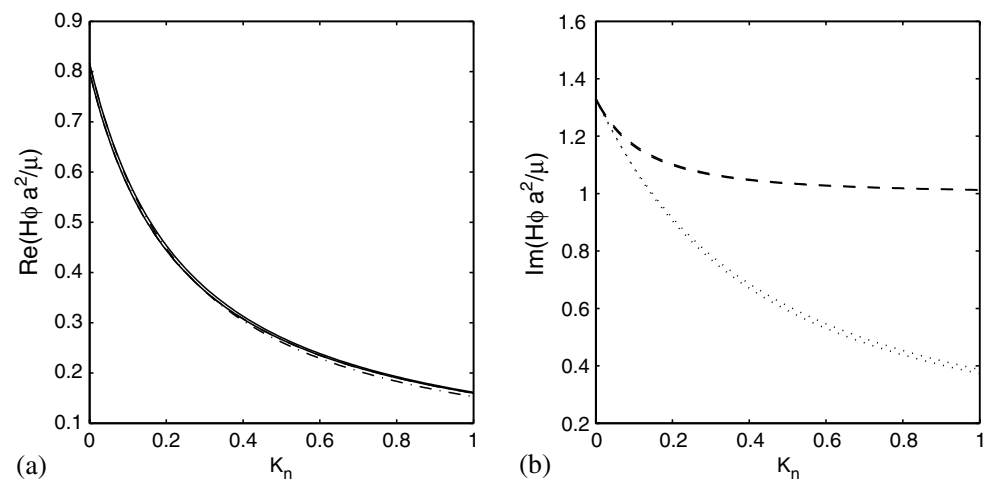

Fig. 4. (a) Variations of the real part of $H_{\text {eff }}$ (in plain line) and of $H_{\text {app }}$ (in dash-dot line) with respect to the Knudsen number for two values of the pulsation $\left(w^{*}=0.5\right.$ and $\left.w^{*}=5\right)$. (b) Variations of the imaginary part of $H_{\text {eff }}$ (in dashed line) and $H_{\text {app }}$ (in dot line) with respect to the Knudsen number for the same two values of the pulsation.

\section{Conclusion}

We have derived the dynamic filtration law that describes the acoustics with wall-slip flow of a gas saturated rigid porous medium. The most general law differs from the generalized Darcy's law in the fact that the effective dynamic permeability depends on the Knudsen number. We have shown that at low Knudsen number, the dynamic permeability could be approximated by the generalized Klinkenberg's law. Analysis of these results on the simple geometry of a bundle of capillaries has led to the conclusion that the generalized Klinkenberg's law fails at reproducing the inertial effect at high pulsation. Further developments of the present work should include computation and analysis of the dynamic permeability on more complex geometries.

\section{References}

Auriault, J.-L., 1980. Int. J. Eng. Sci. 18, 775-785.

Auriault, J.-L., 1991. Int. J. Eng. Sci. 29 (7), 785-795. 
Benssoussan, A., Lions, J.-L., Papanicolaou, G., 1978. Asymptotic Analysis for Periodic Structures. North-Holland Publishing Company, Amsterdam.

Biot, M.A., 1956. J. Acoust. Soc. Am. 28 (2), 168-191.

Burridge, R., Keller, J.B., 1981. J. Acoust. Soc. Am. 70, 1140-1146.

Cercignani, C., 1988. The Boltzmann Equation and its Applications. Springer-Verlag.

Klinkenberg, L.-J., 1941. Drilling and Production Practice. American Petroleum Inst. pp. 200-213.

Levy, T., 1979. Int. J. Eng. Sci. 17, 1005-1014.

Sanchez-Palencia, E., 1980. Lecture Notes in Physics. Springer-Verlag, Berlin. p. 127.

Skjetne, E., Auriault, J.-L., 1999. Transp. Porous Media 36, 293-306. 